\title{
Effect of cholesterol concentration on size of liposome
}

\author{
${ }^{1}$ Sarvesh Kumar Pathak ${ }^{*},{ }^{2}$ Roli Mishra ${ }^{*}{ }^{3}$ Sapan Kumar ${ }^{*},{ }^{4}$ Gyan Prakash, and \\ ${ }^{5}$ Dr. R. Parthasarthy \\ ${ }^{1,2, \& 3}$ B.Pharm Final year students Faculty of Pharmacy, Kamla Nehru Institute of Management and \\ Technology, Sultanpur, Utter Pradesh India, \\ ${ }^{4}$ Faculty of Pharmacy, Kamla Nehru Institute of Management and Technology, Sultanpur, Utter Pradesh, India. \\ ${ }^{5}$ Director of Kamla Nehru Institute of Management and Technology, Faculty of Pharmacy Sultanpur.
}

\begin{abstract}
In this article information about effect of cholesterol concentration on vesicle size of liposome. The advantages and disadvantages of the methods have been described in terms of size distribution and encapsulation efficiency. The reduction of the size of the multilamellar vesicles (MLVs) to small unilamellar vesicles (SUVs) so as to increase their plasma lifetime and consequently increase the possibility of achieving greater tissue localisation.
\end{abstract}

Key words - Liposomes, phospholipid \& encapsulation.

\section{INTRODUCTION}

Liposome was discovered about 40 years ago by Bangham and co-workers and was defined as microscopic spherical vesicles that form when phospholipids are hydrated or exposed to an aqueous environment. ${ }^{1}$ Liposomes are microscopic vesicles composed of a bilayer of phospholipids or any similar amphipathic lipids. They can encapsulate and effectively deliver both hydrophilic and lipophilic substances, ${ }^{2,3}$ and may be used as a non-toxic vehicle for insoluble drugs. ${ }^{4}$ Weiner et $\mathrm{al}^{5}$ defined liposome as a microstructure consisting of one or more concentric spheres of lipid bilayer separated by water or aqueous buffer compartments (Fig.1).

The typical characteristic of bilayerforming lipids is their amphiphilic nature: a polar head group covalently attached to one or two hydrophobic hydrocarbon tails. When these lipids, e.g., phosphatidylcholine, phosphatidyl ethanolamine or phosphatidyl glycerol, are exposed to an aqueous environment, interactions between themselves (hydrophilic interactions between polar head groups and van der Waals interactions between hydrocarbon chains and hydrogen bonding

with water molecules) lead to spontaneous formation of closed bilayers. ${ }^{6}$ Liposomes can differ in size, ranging from the smallest vesicle (diameter $20 \mathrm{~nm}$ ) to liposomes that are visible under the light microscope, with a diameter of $1 \mu \mathrm{m}$ or greater, equal to the dimensions of living cells. ${ }^{6}$ Liposome can carry drugs in one or three potential compartments (water soluble agents in the central aqueous core, lipid soluble agents in the membrane, peptide and small proteins at the lipid aqueous interface). They are classified structurally into multilamellar vesicles (MLVs) and unilamellar vesicles (ULVs). ${ }^{7}$ ULVs have a single phospholipid bilayer membrane and a diameter of $0.05-0.25 \mu \mathrm{m}$. These liposomes (i.e., ULVs) can be further classified into large unilamellar vesicles (LUVs) with a diameter of $0.05-0.25 \mu \mathrm{m}$ and small unilamellar vesicles (SUVs) with a diameter of $0.05-$ $0.10 \mu \mathrm{m}$.

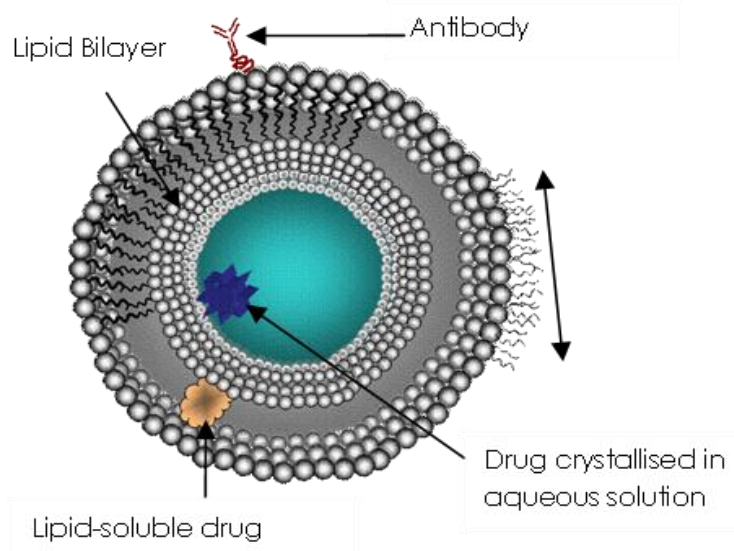

Fig.1- Structure of Liposome

www.iosrjournals.org 
Liposomes are designed in such a way that the solute can be entrapped in the aqueous compartment (polar solute) or embedded in the lipid bilayers (lipohilic or amphiphilic solute). Since ULVs contain a large central aqueous compartment, they are ideally suited for the encapsulation of water soluble agents. ${ }^{8}$ MLVs are composed of concentric phospholipid bilayer membranes in an onion skin arrangement and have a diameter of $1-5 \mu \mathrm{m}$. ULVs contain a small aqueous compartment $(<10 \%)$, which means that they preferentially entrap lipid soluble drugs. ${ }^{8}$ This observation is important to the design of liposomes for cancer therapy since a number of active cytotoxic drugs are highly lipid soluble. ${ }^{9}$

Liposomes have been studied for many years as carrier systems for drugs, ${ }^{10}$ with advantages such as enhancement of therapeutic efficacy at low dosage and, hence, reduction in toxicity of the encapsulated agent; improved pharmacokinetic profiles, e.g., enhanced tissue penetration and increased biological half life; targeting to tumour tissues, e.g., liposomal doxorubicin; and increased stability of the drug particularly against enzymatic degradation. ${ }^{2,11,12}$ The purpose of this study is to review current research efforts in the technology of liposome production and to review current biomedical applications of liposomes, with a view to stimulating further research in this area of study. Literature search was the major approach for the study.

\section{MATERIAL AND METHODS}

Methanol, Chloroform, Ethanol, Potassium dihydrogen Phosphate, Disodium hydrogen Phosphate, Cholesterol and Egg Phosphatidylcholine.

\section{PREPARATION OF PHOSPHATE BUFFER SOLUTION pH 6.8}

Dissolve $28.80 \mathrm{gm}$ disodium hydrogen phosphate \& $11.45 \mathrm{gm}$ of potassium dihydrogen phosphate in $20 \mathrm{ml}$ of distilled water in the $1000 \mathrm{ml}$ volumetric flask, make up the volume up to $1000 \mathrm{ml} \&$ maintain the pH 6.8 (Indian Pharmacopoeia 1996).

\section{FORMULATION OF LIPOSOME}

This is basically a transcription of the procedure outlined in the pink book (pp. 37-38), with a few comments of my own.

i) Make up the lipid mixture containing $100 \mathrm{mg}$ of egg PC, $40 \mathrm{mg}$ of cholesterol and $10 \mathrm{mg}$ of phosphatidil glycerol (PG), in $5 \mathrm{ml}$ of chloroform/methanol solvent mixture $(2: 1 \mathrm{vol} / \mathrm{vol})$.

The lipid mixture suggested here seems to have too much cholesterol. In fact, if one looks at the molar ratio it turns out that there's almost one molecule of cholesterol per molecule of egg PC. This high ratio might make the phase transition disappear, as is described in chapter 1 of the pink book. A seemingly more sensible ratio could be used, following Nevaux's paper, is 6:3:1 egg PC cholesterol: PG. I add the three powders to the empty $100 \mathrm{ml}$ flask, add the chloroform $(3.3 \mathrm{ml})$

and then the methanol $(1.7 \mathrm{ml})$.

ii) Introduce the lipid solution into a $250 \mathrm{ml}$ round bottom flask with a ground glass neck.

iii) Attach the flask to a rotary evaporator, evacuate, rotate about 60rpm and immerse the flask in a thermos tatted water bath set at $30 \mathrm{C}$ (or above the transition temperature of the lipids used)

The powder doesn't seem to dissolve readily in the chloroform/methanol solution. Hence it turned out to be good ideas to le the flask rotate over the water bath (at a temperature above the transition temperature of the lipids which is $\sim 41 \mathrm{C}$. I set it at $50 \mathrm{C}$ ) for about half an hour before starting the vacuum pump. A very low nitrogen flux could be set up to prevent too much oxygen to get dissolved. If this flux is too high the solvent will then evaporate. I set the nitrogen flux thorough the inlet tap, so it goes directly into the flask. Some of the solvent evaporates inevitably during this time, but the solution thermalizes and the lipids dissolve. Only after this time the vacuum pump should be turned on, together with the cooling coil. Allowing cold water to flow through the coil before turning on the vacuum results in water condensating inside evaporator. Placing a clamp on the corresponding hose controls the amount of vacuum. The vacuum was set usually above 10inHg, around $15 \mathrm{inHg}$. This can be further controlled by the nitrogen flux. Again, a high nitrogen flux will result in a too rapid evaporation of the solvent, an undesired situation given that one wants a uniform film to be deposited on the walls of the flask.

iv) Continue until all the liquid has evaporated from solution, and a dry lipid film has been deposited on the walls of the flask. 
v) Continue for 15 min after the dry residue first appears.

At some point, when most of the solvent has evaporated, bubbles start forming on the bottom of the flask. At low vacuum $(<10 \mathrm{inHg})$ these bubbles are many and small. For high vacuum $(\sim 20 \mathrm{inHg})$ a big portion of the film detaches from the bottom of the flask, and forms a big bubble which eventually breaks.

\section{SIZING}

Liposomes are sized using the Dynamic Light Scattering apparatus in the Central Drug Research Institute Lucknow, Utter Pradesh, India.

\section{RESULT}

Liposomes are sized using the Dynamic Light Scattering apparatus .The size of Liposome at different concentrations of Phosphatidylcholin and Cholesterol are tabulated in table-1.

TABLE -1- Effect of Cholesterol Concentration on vesicle size of Liposome

\begin{tabular}{|c|c|c|c|c|}
\hline S.No. & $\begin{array}{c}\text { Formulation } \\
\text { Code }\end{array}$ & $\begin{array}{c}\text { Amount of } \\
\text { Cholesterol (ml) }\end{array}$ & $\begin{array}{c}\text { Amount of } \\
\text { Phosphatidylcholine (ml) }\end{array}$ & $\begin{array}{c}\text { Size of Liposome } \\
(\mathrm{nm})\end{array}$ \\
\hline 1. & LP1 & 30 & 30 & 1625 \\
\hline 2. & LP2 & 40 & 60 & 1323 \\
\hline 3. & LP3 & 60 & 40 & 321.3 \\
\hline 4. & LP4 & 70 & 30 & 207.5 \\
\hline 5. & LP5 & 80 & 20 & 198.1 \\
\hline
\end{tabular}

Key point- LP1, LP2, LP3, LP4 \& LP5 - Formulated Liposome 1, 2, 3, 4, \& 5 respectively

In LP1 the amount of cholesterol is lesser $(30 \mathrm{ml})$ then the vesicle size of liposome is larger but in LP5 the amount of cholesterol is grater $(80 \mathrm{ml})$ then the vesicle size of liposome is smaller.

The effect of cholesterol concentration on vesicle size of Liposome is tabulated in graph1.

\section{Graph 1 - Effect of Cholesterol concentration on vesicle size of Liposome}

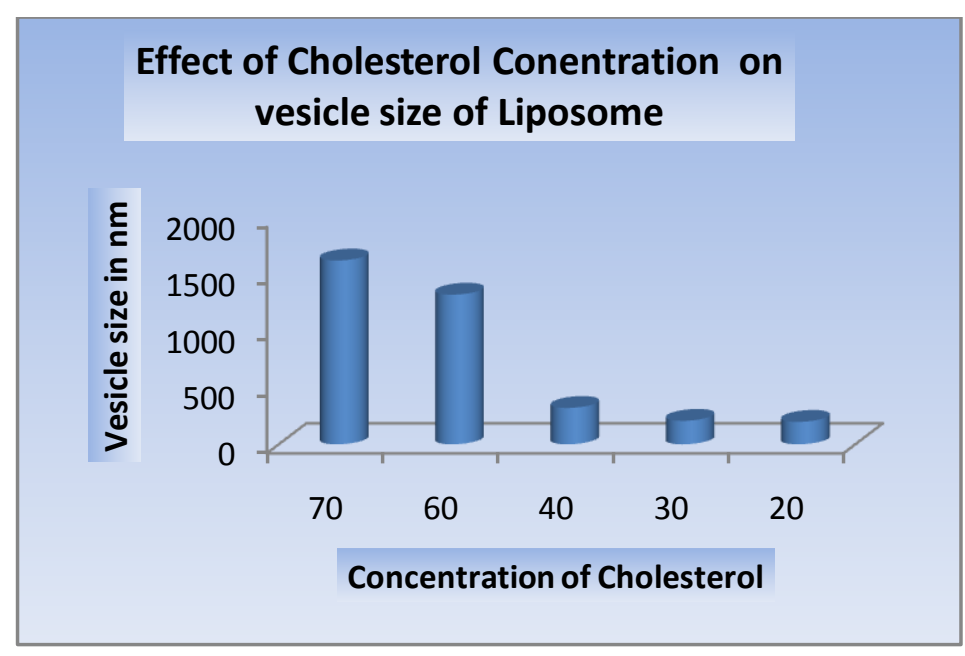

CONCLUSSION

As the ratio of cholesterol is changed the vesicle size of the liposome is changes. The quantity of Cholesterol is increased then the vesicle size is reduced. 


\section{ACKNOWLEDGEMENT}

Authors express their sincere thanks to Mr. Gyan Prakash Assistant professor, Department of Pharmaceutics, Kamla Nehru Institute of Management and Technology Sultanpur India for guidance and Dr. R. Parthasarthy Director of Kamla Nehru Institute of Management and Technology Sultanpur, India, for providing research facilities in the laboratories of the institute and his constants encouragement to carry out the research work.

\section{REFERENCES}

[1] Bangham AD, Standish MM and Watkins JC.Diffusion of univalent ions across the lamellae of swollen phospholipids. $J$ Mol Biol 1965; 13: 238-252.

[2] Fielding MR. Liposomal drug delivery: advantages and limitations from a clinical pharmacokinetics and therapeutic perpective. Clin Pharmacokinet 1991; 21: 155-164.

[3] Akbarieh M, Besner JG, Galal A and Tawashi R. Liposomal delivery system for the targeting and controlled release of praziquantel. Drug Dev Ind Pharm 1992; 18: 303-317.

[4] Lidgate DM, Felgner PL, Fleitman JS, Whatley J and Fu RC. Invitro and invivo studies evaluating a liposome system for drug solubilisation. Pharm Res 1988; 5: 759-764.

[5] Weiner N, Martin F and Riox M. Liposomes as drug delivery system. Drug Dev Ind Pharm 1989; 15(10): $1523-1554$.

[6] Frezard F. Liposomes: from biophysics to the design of peptide vaccines. Braz J Biol Res 1999; 32(2): 181-189.

[7] Perez-Solar R. Liposomes as carriers of antitumor agents: toward a clinical reality.Cancer Treat Rev 1989; 16 : 67-82.

[8] Harrington KJ, Konstantinos N and Richard GV. Liposomal targeted cytotoxic drugs for the treatment of cancer. $J$ Pharm Pharmacol 2002; 54: 1573-1600.

[9] Leyland-Jones B. Targeted drug delivery. Semin Oncol 1993; 20: 12-17. 10. Storm G and Crommelin DJA. Liposomes: quo vadis. Pharm Sci Tech Today 1998; 1: 19-31.

[10] Gregoriadis G. Overview of liposomes. J Antimicrob Chemother 1991; 28(supp. B): 39-48.

[11] Xian-rong Q, Yoshie M and Tsuneji N. Effect of soybean-derived sterols on the in vitro stability and the blood circulation of liposomes in mice. Int J Pharm 1995; 114: 33-41. 